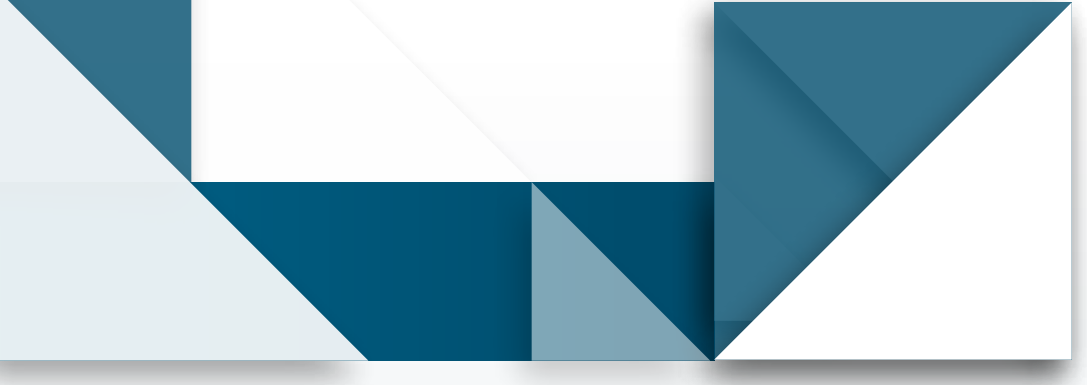

\title{
Representaciones infantiles sobre las notaciones
} numéricas

\section{- Children's representations about numeric notations}

- Representações infantis sobre as notações numéricas

\section{Resumen}

Los adultos alfabetizados estamos habituados a interpretar y producir escrituras numéricas; esta utilización no siempre conlleva una reflexión sobre el proceso de construcción alcanzado por el hombre en el transcurso de su historia. El sistema de numeración escrito es considerado como un lenguaje universal que se utiliza a diario en explicaciones, comunicaciones, en heterogéneas situaciones individuales y sociales. Por más que los niños se encuentren con esta herencia cultural, no escapan a la construcción cognitiva de comprender, producir y utilizar estas escrituras convencionalmente. La educación formal tiene un lugar privilegiado para considerar la enseñanza de estos conocimientos, desde las primeras instancias educativas continuándose en los sucesivos niveles escolares.

La enseñanza formal, aun tratándose de sujetos muy pequeños, es la que tiene la responsabilidad de generar un ambiente escolar variado, rico, significativo e incluyente, para que los alumnos puedan construir estos conocimientos culturalmente válidos. Las decisiones e intervenciones didácticas de los docentes serán oportunas para plantear estos desafíos en la enseñanza de la matemática en la educación infantil. Se aspira a que, a través de un proyecto didáctico, los conocimientos infantiles se vinculen con los saberes culturales.

En este artículo se presenta una parte de los resultados de una investigación sobre las representaciones escritas que realizan alumnos de nivel inicial. Si bien los interrogantes que guiaron el estudio fueron cómo los niños realizan sus primeras escrituras numéricas, cómo evolucionan en estos aprendizajes y cómo hacen uso de estos conocimientos sociales; para esta oportunidad se muestran los resultados del primer interrogante mencionado.

Se analizaron variadas escrituras numéricas de niños de 4 y 5 años de escuelas públicas de la ciudad de Neuquén, Argentina. Se observó que los niños muestran maneras particulares de registrar una determinada cantidad; algunos realizan marcas pictográficas, otros utilizan icónicos y a medida que van creciendo usan mayoritariamente el símbolo numérico convencional. El conocimiento construido es un aporte para la formación docente y para los profesores interesados en la enseñanza del número y la numeración escrita en sujetos que transitan estos primeros aprendizajes.

\section{Adriana Marisa Cañellas* María Josefa Rassetto**}

Palabras clave:

Nivel inicial, representaciones gráficas, notaciones numéricas, enseñanza, aprendizaje.
Universidad Nacional del Comahue. Argentina: fiorentina17@gmail.com

** Universidad Nacional del Comahue. Argentina: mirassetto@hotmail.com 


\section{Abstract}

Literate adults are used to interpret and produce numerical writings; this use does not always entail a reflection on the process of building reached by the man over the course of its history. The Written numerical system is considered to be a universal language that is daily used in explanations, communications, in heterogeneous social and individual situations. Even though children have this cultural heritage, they do not escape the cognitive construction of understanding, production and use of this writing. Formal education is a privileged place to consider the teaching of knowledge, since early educational stages to the higher ones.

Formal education, even in the case of children, is the one that has the responsibility of creating a varied, rich, meaningful and inclusive school environment so that students can build this culturally valid knowledge. Decisions and educational interventions of teachers will be appropriate to consider these challenges in the teaching of mathematics in early childhood education. This project is aimed at linking children's knowledge with cultural knowledge.

This article presents some of the results of a research on written representations made by early stage students. The questions guiding the study were how children make their first numerical writings; how they evolve in this learning; and how they use these social skills. This paper presents the results of the first mentioned question.

Varied numerical writings by four-and five-year old children were analyzed in public schools of Neuquen city in Argentina. It was observed that children show particular ways to register a certain amount; some of them make pictographic marks, others use iconic marks and as they grow, they mostly use the conventional numerical symbol. The constructed knowledge is a contribution to the teacher's formation and to the in-service professors who are interested in the teaching of numbers and the written numeration in individuals that are going through this first learning.

\section{Keywords:}

Early stages, graphic representations, numerical notations, teaching, learning. 


\section{Resumo}

Os adultos alfabetizados estamos acostumados a interpretar e produzir escrituras numéricas; esta utilização nem sempre implica em uma reflexão sobre o processo de construção alcançando pelo homem no transcurso da sua história. $\bigcirc$ sistema de numeração escrito é considerado uma linguagem universal que se utiliza diariamente em explicações, comunicações, em heterogêneas situações individuais e sociais. Por mais que as crianças se encontrem com essa herança cultural, não escampam à construção cognitiva de compreender, produzir e utilizar estas escritas convencionalmente. A educação formal é um lugar privilegiado para considerar o ensino destes conhecimentos, desde as primeiras instâncias educativas e, em continuidade, pelos sucessivos níveis escolares.

O ensino formal, mesmo se tratando de sujeitos muito pequenos, é o que possui a responsabilidade de gerar um ambiente escolar variado, rico, significativo e inclusivo para que os alunos possam construir estes conhecimentos culturalmente válidos. As decisões e intervenções didáticas dos docentes serão oportunas para abordar estes desafios no ensino da matemática na educação infantil. Espera-se que através de um projeto didático, os conhecimentos infantis se vinculem com os saberes culturais.

Neste artigo se apresenta uma parte dos resultados de uma investigação sobre as representações escritas que realizam alunos de nível inicial. Como as questões norteadoras deste estudo foram como as crianças realizam suas primeiras escritas numéricas, como evolvem nestas aprendizagens e como fazem uso destes conhecimentos sociais, para esta oportunidade, se apresentam os resultados do primeiro questionamento mencionado.

Foram analisadas diversas escritas numéricas de crianças de quatro a cinco anos de escolas públicas da cidade de Néuquen, Argentina. Se observou que as crianças apresentam maneiras particulares de registrar uma determinada quantidade; alguns fazem marcas pictográficas, outros utilizam ícones e, a medida em que vão crescendo, usam majoritariamente o símbolo numérico convencional. $\bigcirc$ conhecimento construído é um aporte para a formação docente e para os professores interessados no ensino do número e a numeração escrita para sujeitos que transitam nestas primeiras aprendizagens.

Palavras-chave:

Nível inicial, representações gráficas, notações numéricas, ensino, aprendizagem. 


\section{Introducción}

Desde hace unos años, en Argentina, la educación matemática en el nivel inicial ${ }^{1}$ transita un camino en el cual los niños pequeños pueden acceder al significado de los contenidos matemáticos a través de una actividad intelectual (Charlot, 1986). El trabajo compartido implica, por un lado, que los niños puedan confrontar sus ideas, aceptar errores, valorar su propio trabajo o el ajeno, adaptarse a las restricciones que le presente una situación particular, recomenzar la búsqueda en función de los aportes individuales y grupales; y por otro lado, un docente que pueda reconocer los saberes de los niños, plantear situaciones problemáticas significativas, trabajar a partir de la diversidad de sus alumnos, diseñar propuestas que favorezcan el desarrollo de la autonomía de los niños.

Moll (1990) destaca la importancia que reviste enfrentar a los niños pequeños a diversos escenarios donde tengan la necesidad de resolver situaciones involucrando sus conceptos cotidianos en el campo de los conocimientos científicos, ya que estos se encuentran interconectados y son interdependientes. De esta manera a través de la utilización de conceptos cotidianos, los niños logran otorgarles sentido a los conceptos científicos. Admitiendo esta idea, resulta prioritario analizar el lugar que ocupan los conceptos científicos en la configuración de las nuevas prácticas de enseñanza.

La continuidad y diversidad de las propuestas del docente enriquecen el patrimonio conceptual de los alumnos, incentivando en cada oportunidad la reflexión y el análisis de las situaciones planteadas. Visto de esta forma, es necesario considerar que la escuela, en una sociedad organizada, tiene un rol muy importante para el desarrollo de los procesos psicológicos superiores, porque es en la escuela donde se abordan los aprendizajes intencionales, diferentes de los aprendizajes espontáneos que el niño construye en su relación con el medio. Tanto la educación formal como la informal son instrumentos de enculturación del niño. Kohl de Oliveira (2004, pp. 49) afirma:

En el marco de la teoría sociocultural, la transmisión de los saberes considerados relevantes por un grupo social en un momento histórico determinado queda en manos, en buena parte, de las prácticas educativas intencionales. Es decir, es la escuela, como institución social, la responsable de esta apropiación por parte de los sujetos. Estos saberes son considerados como los "saberes a enseñar" e incluyen fundamentalmente el sistema de escritura y las nociones científicas.

Los niños no solo aprenden en el ámbito escolar; en su contexto social elaboran conocimientos espontáneos, los cuales deben ser complejizados, modificados, ampliados en el entorno escolar y aunque el niño emplee diaria- 
mente estos aprendizajes, con frecuencia muy imprecisos e inestables, no ha desarrollado los conceptos científicos correspondientes. Pero esto no le impide usarlos en contextos concretos y resolver situaciones problemáticas que están a su alcance. Será, pues tarea del docente proponer y ampliar estos contextos de uso, y enfrentarlos a diversas situaciones problemáticas.

Teniendo en cuenta que un concepto no se aprende en una lección, ni resolviendo varias actividades -se trata de un largo camino que recorre el alumno a lo largo de su escolaridad otorgándole diversos significados y sentidoses que nos proponemos, desde hace ya varios años, investigar sobre la enseñanza y el aprendizaje de conceptos científicos en niños pequeños (Rassetto y Cañellas, 2008, 2009; Cañellas y Rassetto, 201 1). En este trabajo en particular se investigó sobre las representaciones escritas que los niños ponen en juego ante la resolución de determinados problemas que involucran escrituras numéricas y sobre la evolución de estas representaciones, mostrando así sus aprendizajes sobre este sabermatemático.

\section{Las notaciones numéricas}

Son numerosas las investigaciones sobre las notaciones numéricas; algunas se inclinan más por conocer cómo el niño las comprende desde un punto de vista psicológico, como lo han hecho Aglioy Martini (1995); Hughes (1987); Martí (1999, 2006); Sastre y Moreno (1980); Schever, Bressan y Merlo de Rivas (2001); Schever, Sinclair, Merlo de Rivas y Tiéche Christinat (2000); Sinclair, Siegrist y Sinclair (1982); Sinclair y Sinclair (s.f.); Tolchinsky (1995). Algunos de estos estudios señalan que los niños tienen que recorrer un largo camino en la construcción de estos conocimientos, en el cual van progresando desde marcas no sistemáticas, pasando por representaciones pictográficas e icónicas hasta llegar al símbolo numérico. Otras publicaciones hacen hincapié en cuestiones que tienen que ver con la génesis histórica de la construcción del número y de los sistemas de numeración por parte de la humanidad, como lo hicieron Conant (1994); Dantzing (1971); Guedi (1998); Ifrah (2008); Smithy Ginsburg (1994).También encontramos investigaciones donde el interés se encuentra puesto en la enseñanza y el aprendizaje de estos conceptos en el marco escolar, como Brissiaud (1993); Lerner (2000); Lerner, Sadovsky y Wolman (1994); Sastre y Moreno (1980); y Terigi y Wolman (2007).

Es de destacar la importancia de los sistemas externos de representación en el desarrollo de la inteligencia humana. Los humanos poseen la capacidad para dejar marcas permanentes de manera intencional para dar a conocer una información usando un sistema de representación externo. Según Martí (2006), en este concepto encontramos la escritura alfabética, las notaciones musicales, el dibujo, las fotografías, los mapas, la notación numérica, las imágenes, las fotografías, los diagramas, los modelos a escala o los medios informáticos.

También, Alvarado y Brizuela (2006) hacen una diferenciación entre el dibujo y los sistemas de escritura convencionales. Estas autoras sostienen que si bien las marcas gráficas intencionales son características del hombre, los sistemas gráficos corresponden a una naturaleza especial, que se diferencia de otras manifestaciones pictóricas o gráficas, y afirman:

Mientras que dibujar es una actividad gráfica individual que, en la tradición occidental actual, no es restrictiva, en el sentido que no condiciona el empleo de elementos específicos, escribir implica enfrentarse con una convención social preexistente que 
demanda ceñirse a sus reglas de composición e interpretación, y a las funciones que socialmente se le han otorgado. (Alvarado y Brizuela, 2006, pp. 134)

La construcción de sistemas de representación externos surge de la necesidad comunicativa que desarrolló el hombre. También ha sido necesario realizar notaciones para que el sujeto pueda recordar algo. Es decir, encontramos que las notaciones tienen funciones de comunicar, memorizar y registrar.

Según Schever et al. (2000), los diferentes sistemas convencionales de representación escrita nos aportan herramientas para generar representaciones y comunicarnos en ciertas áreas; además también nos sirven para resolver problemas, crear nuevos objetos, procedimientos e ideas. Es decir, contribuyen a la acción y al aprendizaje en general. Toda representación semiótica tiene una función principal: representar otra realidad (Martí, 2006). A la vez que son objetos materiales, se refieren a otra realidad; por ejemplo, el símbolo numérico remite a la numerosidad de una cierta colección de objetos.

Los símbolos numéricos son considerados sistemas de notaciones. En nuestra cultura podemos encontrar distintas formas de nombrar y de representar externamente un número. Al respecto, Tolchinsky (1995) expresa que los numerales pueden decirse en forma oral, utilizando símbolos lingüísticos; pueden escribirse por medio de palabras escritas; pueden escribirse usando cifras, es decir utilizando el sistema de notación numérica; también pueden representarse gestualmente, por ejemplo, en el lenguaje de señas para personas con discapacidad auditiva.

Dentro de las representaciones matemáticas, el sistema notacional numérico ha logrado una repercusión muy importante desde el punto de vista sociocultural. Este es considerado un lenguaje universal que se utiliza a diario en explicaciones, comunicaciones, en heterogéneas situaciones individuales y sociales. Pero no solo su valor se encuentra en su presencia en los procesos históricos y sociales, sino también por ser un objeto de conocimiento que debe ser enseñado para su construcción por parte de los sujetos.

Si bien la presencia en nuestra cultura de las notaciones numéricas es significativa, es muy habitual considerarlas como algo natural y no se repara en el largo y dificultoso camino que se recorrió para lograr la construcción con la que contamos en la actualidad. Es necesario que tengamos en cuenta que los niños también tendrán una ardua tarea en comprender, producir y utilizar nuestro sistema de numeración. Sabemos de obstáculos que tendrán que vencer para representar una determinada cantidad de una colección, para recordar esa cantidad, para comunicar de alguna manera esa información a otros, es decir producir e interpretar diferentes representaciones semióticas.

Si consideramos el sistema de numeración escrito desde su uso social, los sujetos se enfrentan en muchas de sus actividades cotidianas a dos tareas muy diferenciadas. Por un lado, se encuentran con la necesidad de interpretar una abundante información con símbolos numéricos, además de participar en co- 
mentarios sobre los mismos; por otra parte, son ellos mismos los que precisan usar esos símbolos escritos para comunicar algo. Leer y escribir números no son actividades recíprocamente inversas, como tampoco lo son la escritura y la lectura en general. En síntesis, no es lo mismo que los niños pequeños interpreten una notación numérica a que representen una cierta cantidad o un determinado ordenamiento. Estas ideas servirán para fundamentar decisiones en un contexto didáctico.

\section{Representaciones numéricas realizadas por niños de 4 y 5 años}

En esta investigación se analizan y se reflexiona sobre las escrituras de los alumnos ${ }^{2}$ de salas de 4 y 5 años, a partir delos diversos modos de representación que usan cuando resuelven tareas de registros de cantidades, es decir cuando los niños tienen necesidad de utilizar el sistema de notaciones numérico.

Con respecto a estos modos de representación gráfica, estudios anteriores dan cuenta de los resultados hallados al clasificar las producciones numéricas infantiles. A continuación mostramos las categorizaciones utilizadas en algunas investigaciones precursoras, según los tipos de registros:

- Sastre y Moreno (1980): dibujo sin ninguna relación con el número; dibujos o esquemas en correspondencia biunívoca con cada uno de los objetos; tantas cifras como objetos, grafismo correcto.

- Sinclair, Siegrist y Sinclair (1982): representación global de la cantidad;

2 Se trabajó con 47 niños de escuelas públicas de la ciudad de Neuquén, Argentina. Los registros recolectados para su análisis fueron $87 ; 51$ de sala de 5 años y 36 de sala de 4 años. representación de la clase de objetos; correspondencia uno a uno sin numerales; correspondencia uno a uno con numerales; solo el valor del cardinal y valor cardinal y clase de objetos.

- Hughes (1987): representaciones idiosincrásicas; pictográficas; icónicas; simbólicas.

- Brissiaud (1993): representaciones analógicas de las cantidades (colecciones de muestra); números convencionales.

- Aglio y Martini (1995): imagen-garabato; dibujo de objetos; signos; dibujo de los dedos de la mano; símbolos numéricos personales, símbolos numéricos convencionales; numerales convencionales.

A partir de estos antecedentes, y del análisis de los resultados obtenidos en nuestra investigación, se construyeron las siguientes categorías:

Respuestas idiosincrásicas: dejan marcas en la hoja, pero no remiten a algún significado que se pueda interpretar.

Respuestas pictográficas: realizan dibujos lo más parecidos posibles a los objetos que están cuantificando, repitiéndolos tantas veces según sean los objetos a través de una correspondencia uno a uno.

Respuestas icónicas: usan un símbolo de diseño propio (generalmente muy simple), el cual va a representar cada objeto en cuestión, haciendo una correspondencia uno a uno.

Respuestas usando el símbolo numérico:

- Repetición de un mismo símbolo numérico: escriben el símbolo numérico correspondiente al cardinal de la colección tantas veces como la cantidad a representar. 
- Escritura de la serie numérica ordenada: escriben varios números ordenados, generalmente lo hacen desde el uno. Hacen tantos como la cantidad a representar.

- Escritura del símbolo y su representación icónica: dejan por escrito el número correspondiente y también hacen tantos iconos como indica la cantidad de objetos.

Respuestas simbólico-convencionales:

- Uso del símbolo de manera convencional: utilizan correctamente el símbolo numérico convencional para representar la totalidad de objetos.

- Notaciones mixtas: además del símbolo numérico correcto, hacen referencia a la clase de objetos que están cuantificando, ya sea con palabras o dibujos.

Los resultados encontrados se registran en la tabla 1. Allí se puede comparar los tipos de representaciones que realizaron los niños de cada edad, de acuerdo conla categorización usada:

Tabla 1. Distintos tipos de representaciones según niños de salas de 4 y 5 años

\begin{tabular}{ccccccc}
\hline Salas & $\begin{array}{c}\text { Respuestas } \\
\text { idiosincrásicas }\end{array}$ & $\begin{array}{c}\text { Respuestas } \\
\text { pictográficas }\end{array}$ & $\begin{array}{c}\text { Respuestas } \\
\text { icónicas }\end{array}$ & $\begin{array}{c}\text { Respuestas } \\
\text { usando el } \\
\text { símbolo }\end{array}$ & $\begin{array}{c}\text { Repuestas } \\
\text { simbólico- } \\
\text { convencionales }\end{array}$ & $\begin{array}{c}\text { Total } \\
\%\end{array}$ \\
\hline 4 años & 16,6 & 11,1 & 41,8 & 8,3 & 22,2 & 100 \\
\hline 5 años & 5,9 & 7,8 & 25,6 & 11,7 & 49 & 100 \\
\hline
\end{tabular}

\section{Análisis e interpretaciones de las representaciones infantiles}

Al analizar los trabajos realizados por los alumnos fue posible distinguir diferentes modos representativos de una determinada cantidad de objetos. También cabe aclarar que no hubo alumnos que se negasen a realizar la tarea solicitada o que no pudiesen "hacer algo" sobre su hoja. Esta observación también es compartida con Merlo de Rivas, Schevery Criado (s.f.); aunque en otra investigación (Schever et al., 2000), hubo niños que se negaron a producir en un contexto de características clínicas.

A continuación se presentan las categorías utilizadas con el análisis de las producciones infantiles:

Respuestas idiosincrásicas: estas representaciones gráficas son aquellas en las que no se reconoce la información de algún dato numérico. En general, tanto en niños de 4 como de 5 años, son pocos los casos cuyas marcas en el papel son ilegibles para el adulto, donde encontrarle algún sentido sea difícil. Este tipo de representación se halló un 16,6\% en salas de 4 años y un 5,9\% en salas de 5 años. Esta tendencia indica que con el avance de la edad tales marcas sin sentido aparente son cada vez menos frecuentes. En el figura 1 se puede observar un ejemplo de trazos hechos por una niña a los cuales no fue posible asignarles 
algún significado numérico o alfabético. La alumna tenía que anotar la cantidad de peces que había pescado durante un juego.

Figura 1. Marcas dejadas por Micaela, edad: 4 años y 2 meses

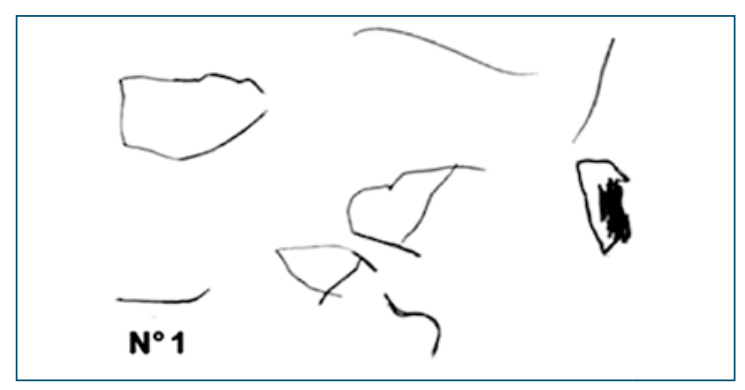

Respuestas pictográficas: en el total de los trabajos analizados, podemos distinguir que, en ambas salas, son muy escasos los alumnos que realizan dibujos similares a los objetos que tienen que cardinalizar. Es posible que los niños encuentren muy difícil realizar uno por uno estos dibujos, solo lo hacen cuando no se han dado cuenta de que con una simple marca pueden representar cada objeto. En salas de 4 años, el $11,1 \%$ realizó estas representaciones con dibujos y en salas de 5 años, el 7,8 \%.

En el caso de Martín (figura 2), observamos cómo dibujó una a una las estrellas que había juntado al cabo de tres tiradas del dado, ante la consigna de anotar el puntaje obtenido. Se evidencia en el dibujo, después de una cantidad de estrellas, que estas no presentan la misma forma, ya no son tan fieles como las primeras.

Figura 2. Estrellas dibujadas por Martín, edad: 4 años 11 meses

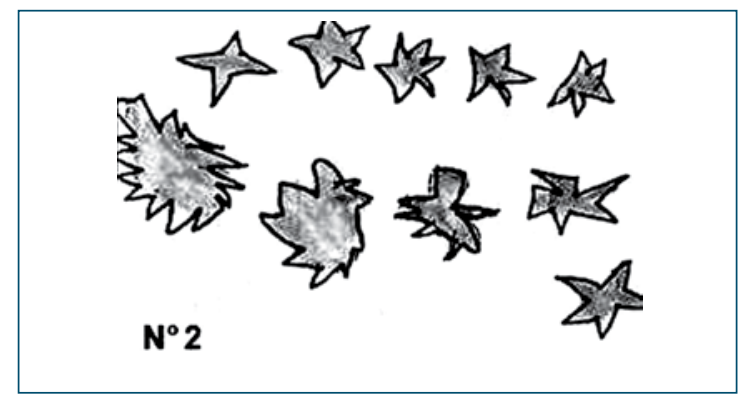

Respuestas icónicas: hemos encontrado este tipo de respuesta en 15 niños (41,8 \%) de sala de 4 años y en $13(25,6 \%)$ de sala de 5 años. Entendemos que es la manera más frecuente de registrar las cantidades para estas edades; y, a medida que se desarrollan van abandonando el uso de estas marcas icónicas y las reemplazan por la notación convencional. Aunque, las marcas brindan la información exacta sobre la cantidad, no hacen referencia a las cualidades de los objetos. Podemos observar a lgnacio haciendo rayitas para representar el puntaje obtenido en el juego de dados (figura 3), estas marcas no se parecen a los objetos involucrados (estrellas).

Figura 3. Rayas hechas por Ignacio, edad: 5 años 3 meses

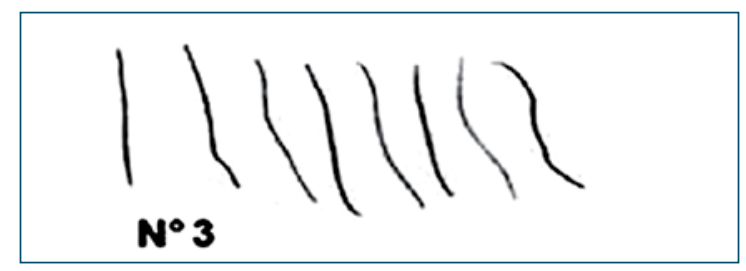

Respuestas usando el símbolo numérico:

- Repetición de un mismo símbolo numérico: escriben el símbolo numérico correspondiente al cardinal de la colección tantas veces como la cantidad a representar.

Estos tipos de registros fueron escasos; en total solo cuatro alumnos $(4,5 \%)$ entre ambas salas recurrieron a estas escrituras. Tal vez los niños que repiten varios símbolos tengan alguna dificultad al tratar de pasar de muchos objetos a un único símbolo; es decir, hay niños que en algún momento pueden pensar que muchos objetos necesitan muchos símbolos para quedar representados por escrito. Según Brissiaud (1993), para el caso de las representaciones numéricas menores a diez objetos, "[...] una pluralidad se representa por un único signo: una pluralidad de ovejas, por ejemplo, 
se representa mediante una sola palabra-número, una sola cifra o un único dedo bajado", nos es tarea sencilla para los niños pequeños darse cuenta es esto.

En el registro de Luli (figura 4), observamos que escribió una cantidad mayor de símbolos " 2 " que la cantidad que tenía que representar, aunque identificó con claridad que dos objetos (los dos peces que había pescado durante el juego) se representan con el símbolo " 2 ", no pudo controlar la cantidad de cifras que debía escribir.

Figura 4. Escritura simbólica de Luli, edad: 5 años, 1 mes

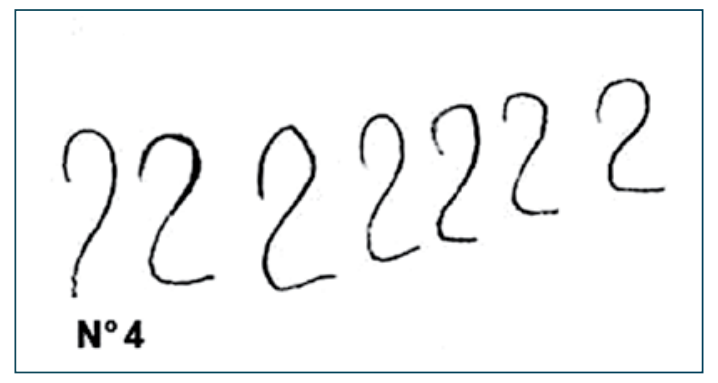

- Escriben varios números ordenados, generalmente lo hacen desde el uno. Hacen tantos como la cantidad a representar.

Yoselín toma el mismo criterio que Luli en cuanto a la repetición de símbolos, pero no utiliza un único símbolo, sino que despliega por escrito la serie numérica ordenada para dar cuenta que así como tiene muchos objetos a representar (los seis peces que pescó), necesita usar muchos símbolos (figura 5).

Figura 5. Escritura simbólica de Yoselín, edad: 4 años, 11 meses

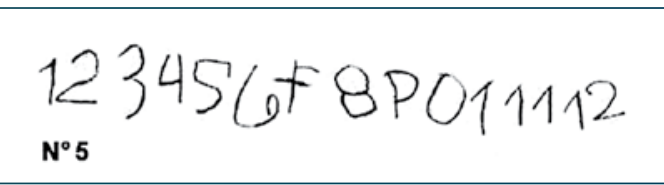

Al analizar estos últimos dos registros nos encontramos con algunas diferencias a lo que Sastre y Moreno (1980) Ilaman "tantas cifras como objetos", y, a su vez, para Sinclair et al. (1982) es el "tipo de notación 4: correspondencia uno a uno con numerales: nuestros numerales son utilizados de dos maneras: se anotan los números del conteo, como '1234' para cuatro pelotas, o el valor del cardinal es anotado tantas veces como su valor, como '4444' para cuatro pelotas".

Tolchinsky (1995, pp. 230), al referirse a escrituras del tipo que analizan las investigadoras anteriores, dice:

Respecto a las cifras, estas son utilizadas para notar la cantidad pero no según las convenciones del sistema notacional ya que repite las cifras tantas veces como sea necesario para corresponder con el número de objetos. Una cifra no basta para mostrar la colección de objetos que intenta representar y por eso recurre a una mayor explicitación. 
Podemos agregar que en esta forma de registrar cantidades, si bien se utiliza el símbolo convencional, las situaríamos en un nivel anterior al uso correcto de la notación numérica. Consideramos que en estos casos se utilizan los símbolos como íconos, es decir, los niños perciben que deben utilizar números escritos ante la consigna de registrar cantidades, no obstante ese conocimiento, no pueden abandonar el procedimiento de repetición de alguna marca que vaya indicando cada objeto. Con respecto a esto, Sinclair et al. (1982, pp. 18) dicen: "finalmente -y este aspecto nos parece importante- bien puede ser que la idea de que cada objeto debe tener un símbolo escrito que le corresponda sea extremadamente poderosa".

- Escritura del símbolo y su representación icónica: dejan por escrito el número correspondiente y también hacen tantos iconos como indica la cantidad de objetos.

Este tipo de registro se encontró muy escasamente, solo el $6 \%$ entre las dos salas. En la figura 6 vemos el trabajo de un alumno que escribe el símbolo "8" y también representa ocho rayas a su costado, indicando los peces obtenidos en el juego.

Figura 6. Escritura simbólica e icónica de Leandro, edad: 5 años, 6 meses

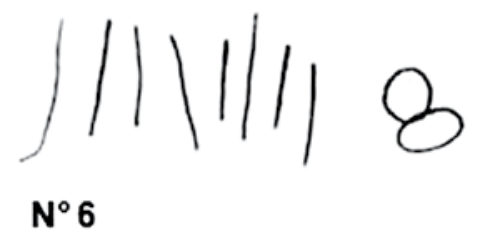

Respuestas simbólico-convencionales:

- Utilizan correctamente el símbolo numérico convencional para representar la totalidad de objetos.
Ocho niños de sala de 4 años pudieron usar convencionalmente el símbolo numérico, es decir, el 22,2\%. En cambio, en la sala de 5 años fue utilizado por el $49 \%$ de los alumnos. Esto indica que estos niños ya han abandonado el registro icónico y pasan a la escritura de las notaciones numéricas convencionales. Como afirman Schever et al. (2000, pp. 212): "con la edad, la producción de notaciones convencionales se hizo más frecuente y se extendió progresivamente a números mayores".

En la figura 7, por ejemplo, observamos la escritura simbólica de Fabricio, quien logró con un solo número representar la totalidad de objetos que obtuvo en el juego de dados.

Figura 7. Escritura simbólica de Fabricio, edad: 5 años, 9 meses

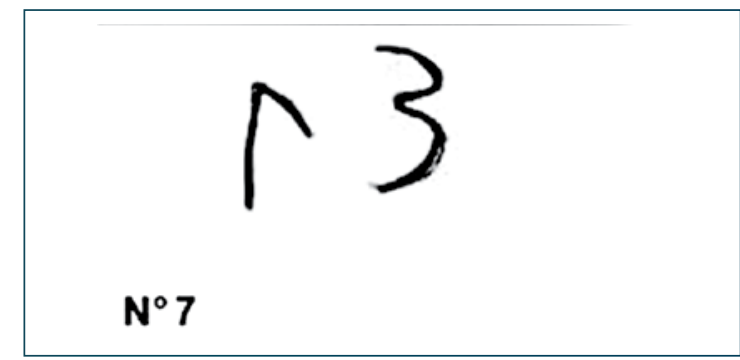

- Notaciones mixtas: además del símbolo numérico correcto, hacen referencia a la clase de objetos que están cuantificando, ya sea con palabras o dibujos.

Una niña, además de registrar correctamente la cantidad de animales de mar que obtuvo en el juego de la pesca, también logró indicar cada clase de estos animales; los diferencia con los dibujos que hace al costado de los símbolos escritos (figura 8). Según Sinclair et al. (1982) en el "tipo de notación 6: valor cardinal y clase de objetos", hay niños que, además del símbolo, se esfuerzan por agregar algo que indique cuál es la clase de objetos con la que están trabajando. En el estudio de Aglio y Martini (1995), Luca, de casi 6 años, 
dibuja el tipo de pieza que necesita y al lado escribe con el símbolo convencional la cantidad. Para Martí (2006), recién a los 7 años los niños pueden usar una doble representación ya que estas implican una diferenciación de las funciones que tienen los dos tipos de registros usados: el de las notaciones numéricas de dar la cantidad de objetos y el dibujo de identificar las clases de objetos.

Figura 8. Escritura simbólica y clases de objetos (pulpos, peces y delfines) de Guadalupe, edad: 6 años, 2 meses

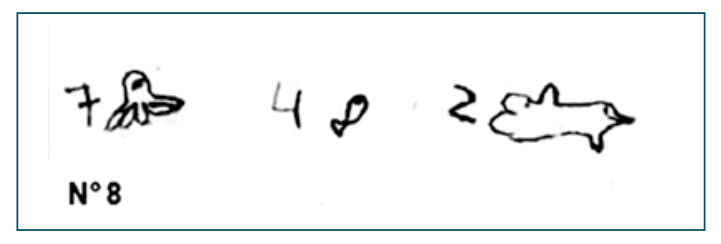

De acuerdo con algunas de las investigaciones anteriores analizadas (Aglio y Martini, 1995; Schever et al., 2000; Martí, 2006), anticipamos la posibilidad de encontrar registros donde los niños escribieran palabras, letras, o letras y números en sus producciones. Schever y Germano (2006, pp. 32) dicen al respecto: "este tipo de producción implica la reutilización de formas notacionales conocidas en otros ámbitos al servicio de la notación de cantidades". Sin embargo, no hubo producciones de ese tipo, ni en salas de 4 ni de 5 años. En el caso de la investigación de Alvarado (2006), tampoco surgieron estos tipos de elaboraciones.

En las producciones de los niños se pueden observar las representaciones analógicas, en las que han simbolizado las cantidades usando correspondencia uno a uno entre cada objeto y una marca, como pueden ser dibujos, rayitas, redondeles, símbolos numéricos repetidos, etc. Estos alumnos, según Brissiaud (1993), no pueden acceder al uso de etiquetas numéricas escritas que simbolizarían al cardinal, sino que necesitan desarrollar una colección de marcas. Con respecto al aprendizaje de estas representaciones Brissiaud afirma: "hay que esperar, por tanto, que la representación de cantidades por una colección de muestra sea más precoz que la representación numérica, por ser más accesible". Por otro lado, nos dimos cuenta que para representar el total de objetos de un conjunto, otros alumnos han utilizado correctamente el símbolo numérico convencional.

Cuando el niño es capaz de realizar representaciones icónicas ya ha realizado un salto cognitivo en sus ideas sobre el número; estas marcas brindan la información exacta sobre la cantidad y no hacen referencia a las cualidades de los objetos. Esto muestra que el niño ya está pensando en que cuando tiene que dejar por escrito cuántos hay, no necesita fijarse en las características cualitativas de los objetos, sino en las cuantitativas.

Coincidimos en los resultados encontrados con las apreciaciones de Schever et al. $(2000,218)$, quienes afirman: 
Las notaciones convencionales aumentan con la edad, pero aun a los 8 años no todas las notaciones son correctas. Los resultados indican que una variedad de ideas numéricas y notacionales intervienen en: la comprensión de la cardinalidad, la secuencia de conteo, las correspondencias entre el modo oral y el modo escrito, las regularidades notacionales, el valor de posición. Las reglas de transcripción se van elaborando lenta y trabajosamente.

Teniendo en cuenta la población de sujetos estudiada y el material bibliográfico consultado, se puede inferir que en primer lugar se dan las representaciones gráficas más primarias para luego dar paso a los registros simbólicos numéricos. Este progreso en la escritura de las notaciones numéricas, creemos que está relacionado con la madurez de los niños, las intervenciones educativas, las experiencias culturales de los sujetos en su ambiente, entre otras causas. Con respecto a la evolución histórica del número, son muchas las investigaciones que afirman que el hombre primitivo pasó del uso de colecciones de muestra con objetos al uso del símbolo numérico convencional, dando cuenta así de su evolución cognitiva (Martí, 2006).

\section{Comentarios finales}

Los resultados nos muestran que el aprendizaje del sistema de numeración escrito es un camino que precisan recorrer los niños; no se trata de un aprendizaje espontáneo, ya que estos conceptos responden a una construcción histórica y cultural. Esto quiere decir que será necesario disponer de estrategias de reconstrucción de determinadas prácticas culturales para que, en correspondencia con el desarrollo cognitivo, los alumnos avancen en sus conceptualizaciones sobre nuestro sistema de numeración decimal.

En cuanto a la responsabilidad de la educación formal, será preciso involucrar a los alumnos en una propuesta didáctica que abarque un encadenamiento de situaciones secuenciadas, no actividades aisladas. Se trata de proponer "una pequeña génesis artificial del concepto" para que el niño construya por sí mismo un conocimiento nuevo (Brousseau, 2007). A diferencia de la génesis histórica, la génesis escolar se produce con una intencionalidad de enseñanza, involucra conocimientos que ya existen en la cultura, toma como referencia la construcción histórica para reconocer los problemas a los que ha dado respuesta el conocimiento que se desea enseñar. Sastre y Moreno (1980, pp. 453) afirman:

Los contenidos de un aprendizaje que no tiene en cuenta la génesis de la adquisición de conocimientos, permanecen como una superestructura impuesta, no integrados al universo de posibilidades de actuación del individuo, rígidamente ligados al contexto en el que fueron aprendidos y no asociados a este.

En la medida que se promuevan variadas situaciones donde los niños tengan la necesidad de registrar cantidades y se aliente el intercambio de las diferentes producciones, con sus explicaciones y argumentaciones, se logrará un avance en las conceptualizaciones numéricas de los niños.

Estas contribuciones sobre un pequeño aspecto del pensamiento infantil podrán ser instrumentos para los docentes en su tarea de gestionar en sus alumnos el desarrollo del pensamiento conceptual. 


\section{Referencias Bibliográficas}

Aglio, F. y Martini, A. (1995). Rappresentazione e notazione Del La quantità in ETA prescolare. Età evolutiva, 51, 30-44.

Alvarado, M. (2006). La representación gráfica de cantidades discretas. Entre las posibilidades infantiles y las restricciones de la tarea. En: M. Alvarado y B. Brizuela (comps.). Haciendo números. Las notaciones numéricas vistas desde la psicología, la didáctica y la historia, (pp- 91 -108). Buenos Aires: Paidós.

Alvarado, M. y Brizuela, B. (comps.). (2006). Haciendo números. Las notaciones numéricas vistas desde la psicología, la didáctica y la historia. Buenos Aires: Paidós.

Brissiaud, R. (1993). El aprendizaje del cálculo. Más allá de Piaget y de la teoría de conjuntos. Madrid: Aprendizaje Visor.

Brousseau, G. (2007). Iniciación al estudio de la teoría de las situaciones didácticas. Buenos Aires: Libros del Zorzal.

Cañellas, A. y Rassetto, M. (201 1). Magnitudes y medidas. Aportes para la educación infantil. Neuquén: Editorial Educo.

Charlot, B. (1986). La epistemología implícita en las prácticas de enseñanza de las matemáticas. Capítulo traducido por Bkouche, Charlot y Rouche. Faire des Mathématiques: le plaisir du sens. Cannes, Francia: Editorial ArmandColin.

Conant, L. (1994). El arte de contar. En: J. Newman. El mundo de las matemáticas, (pp. 134). Tomo 4. Barcelona: Editorial Grijalbo.

Dantzing, T. (1971). El número lenguaje de la ciencia. Buenos Aires: Sudamericana S. A. Guedi, D. (1998). El imperio de las cifras. Barcelona: Ediciones Grupo Zeta.

Hughes, M. (1987). Los niños y los números. Barcelona: Editorial Planeta.

Ifrah, G. (2008). Historia universal de las cifras. Madrid: Espasa-Calpe.

Kohl de Oliveira, M. (2004). Pensar la educación: las contribuciones de Vigotsky. En: J. Castorina, (Ed). Piaget-Vygotsky: contribuciones para replantear el debate, (pp. 45-68). Buenos Aires: Piadós Educador.

Lerner, D. (2000). La matemática en la escuela. Aquí y ahora. Buenos Aires: Aique.

Lerner, D., Sadovsky, P. y Wolman, S. (1994). El sistema de numeración: un problema didáctico. En: C. Parra e I. Saiz (comps). Didáctica de la matemática (pp. 218 245). Buenos Aires: Paidós.

Martí, E. (1999). Esto no es un dibujo. Las primeras distinciones sobre sistemas notacionales. En: J. Pozo y C. Monereo. El aprendizaje estratégico, (pp. 239-250). Madrid: Aula XXI. Santillana.

Martí, E. (2006). Las primeras funciones de las notaciones numéricas. Una mirada evolutiva. En: M. Alvarado y B. Brizuela (comp.). Haciendo números. Las notaciones numéricas vistas desde la psicología, la didáctica y la historia, (pp. 51-80). Buenos Aires: Paidós. 
Merlo de Rivas, S., Schever, N. y Criado, N. (s.f.). Caminos...Modalidades en la representación de números. G.I.A.M. Consejo Provincial de Educación de Río Negro. Argentina.

Moll, L. (comp.). (1990). Vygotsky y la educación. Buenos Aires: Aique.

Rassetto, M. y Cañellas, A. (2008). Repensando la enseñanza de conceptos científicos en la educación infantil. Revista Alternativas. Serie Espacio Pedagógico, 12(48), 185-192.

Rassetto, M. y Cañellas, A. (2009). Las conceptualizaciones infantiles sobre las magnitudes físicas y sus medidas. La necesidad del lenguaje en el proceso de enseñanza. En: M. Rassetto y M. Massa (comp.). Ciencias Naturales. Aportes desde la investigación educativa (pp. 345-370). Neuquén: Editorial Educo.

Sastre, G. y Moreno, M. (1980). Descubriendo y construcción de conocimientos. Barcelona: Editorial Gedisa.

Schever, N., Bressan, A. y Merlo de Rivas, S. (2001). Los conocimientos numéricos en niños que inician su escolaridad. En: N. Elichiry (comp.). ¿̇ónde y cómo se aprende? Temas de psicología educacional, (124-137). Buenos Aires: Editorial Eudeba.

Schever, N. y Germano, A. (2006). Conocimientos matemáticos de niños de 4 a 7 años. En: M. Alvarado y B. Brizuela (comps.). Haciendo números. Las notaciones numéricas vistas desde la psicología, la didáctica y la historia. Buenos Aires: Paidós.

Schever, N., Sinclair, A., Merlo de Rivas, S. y Tièche Christinat, C. (2000). Cuando ciento setenta y uno se escribe 10071 : niños de 5 a 8 años produciendo numerales. Revista Infancia, 90, 31-50.

Sinclair, A. y Sinclair, H. (s.f.). Las interpretaciones de los niños preescolares sobre los números escrito. Human Learning, 3, 173-184.

Sinclair, A., Siegrist, F. y Sinclair, H. (1982). Las ideas de los niños pequeños sobre el sistema de numeración escrita. Paper presentado en la Nato Conference on the Acquisition of Symbolic Skill, Universidad de Keele. Inglaterra.

Smith, D. y Ginsburg, J. (1994). De los números a los numerales y de los numerales al cálculo. En: J. Newman. El mundo de las matemáticas (pp. 50- 154). Tomo 4. Barcelona: Editorial Grijalbo.

Terigi, F. y Wolman, S. (2007). Sistema de numeración: consideraciones acerca de su enseñanza. Revista Iberoamericana de Educación, 43, 59-83.

Tolchinsky, L. (1995). Dibujar, escribir, hacer números. En: L. Teberosky y A. Tolchinsky (comps.). Más allá de la alfabetización (pp. 218-239). Buenos Aires: Aula XXI. Santillana.

Vigotsky, L. (1991). El Desarrollo de los Procesos Psicológicos Superiores. México: Editorial Grijalbo. 